\title{
A Novel Hybrid Crossover based Artificial Bee Colony Algorithm for Optimization Problem
}

\author{
Sandeep Kumar \\ Jagannath University \\ Chaksu, Jaipur, Rajasthan, \\ India - 303901
}

\author{
Vivek Kumar Sharma, \\ Ph.D \\ Jagannath University \\ Chaksu, Jaipur, Rajasthan, \\ India - 303901
}

\author{
Rajani Kumari \\ Jagannath University \\ Chaksu, Jaipur, Rajasthan, \\ India - 303901
}

\begin{abstract}
Artificial bee colony (ABC) algorithm has proved its importance in solving a number of problems including engineering optimization problems. $\mathrm{ABC}$ algorithm is one of the most popular and youngest member of the family of population based nature inspired meta-heuristic swarm intelligence method. $\mathrm{ABC}$ has been proved its superiority over some other Nature Inspired Algorithms (NIA) when applied for both benchmark functions and real world problems. The performance of search process of $\mathrm{ABC}$ depends on a random value which tries to balance exploration and exploitation phase. In order to increase the performance it is required to balance the exploration of search space and exploitation of optimal solution of the ABC. This paper outlines a new hybrid of $\mathrm{ABC}$ algorithm with Genetic Algorithm. The proposed method integrates crossover operation from Genetic Algorithm (GA) with original $\mathrm{ABC}$ algorithm. The proposed method is named as Crossover based $\mathrm{ABC}(\mathrm{CbABC})$. The $\mathrm{CbABC}$ strengthens the exploitation phase of $\mathrm{ABC}$ as crossover enhances exploration of search space. The $\mathrm{CbABC}$ tested over four standard benchmark functions and a popular continuous optimization problem.
\end{abstract}

\section{General Terms}

Computer Science, Nature Inspired Algorithms, Metaheuristics.

\section{Keywords}

Artificial bee colony algorithm, Genetic Algorithms, Crossover operator, Travelling Salesman Problem, Particle swarm optimization.

\section{INTRODUCTION}

Nature inspired meta-heuristics has become a looming and interesting field of research among researchers who are working on optimization problems. Almost all meta-heuristics make use of both randomization and local search. Due to randomization it can move away from local search to global search space. That's why meta-heuristics are best suitable for global optimization. Meta heuristic algorithms have two major components: diversification and intensification. Diversification explores the large search space and ensures that solution does not trap in local optima while intensification concentrates on best solution for convergence to optimality [1]. Population based meta-heuristics do not guarantee the optimal solution but they provide near-optimal solution for most difficult optimization problems. Researchers have analyzed such behaviors and designed algorithms that can be used to solve nonlinear and discrete optimization problems. Previous research [2, 3, 4, and 5] has shown that methods based on swarm intelligence have great power to find solutions of real world optimization problems. The algorithms that have emerged in recent years include ant colony optimization (ACO) [2], particle swarm optimization (PSO) [3], bacterial foraging optimization (BFO) [6] etc. Artificial bee colony $(\mathrm{ABC})$ optimization algorithm introduced by $\mathrm{D}$. Karaboga [7] is a new entry in class of swarm intelligence. This algorithm is inspired by the social behavior of honey bees when searching for quality food source. Similar to any other population based optimization algorithm, ABC consists of a population of inherent solutions. The inherent solutions are food sources of honey bees. The fitness is decided in terms of the quality of the food source that is nectar amount. ABC is relatively a simple, fast and population based stochastic search technique in the field of nature inspired algorithms.

There are two fundamental processes which drive the swarm to update in $\mathrm{ABC}$ : the deviation process, which enables exploring different fields of the search space, and the selection process, which ensures the exploitation of the previous experience. However, it has been shown that the ABC may occasionally stop moving toward the global optimum even though the population has not encounter to a local optimum [8]. It can be observed that the solution search equation of $\mathrm{ABC}$ algorithm is good at exploration but poor at exploitation [9]. Therefore, to maintain the proper balance between exploration and exploitation behavior of $\mathrm{ABC}$, it is highly expected to develop a local search approach in the basic $\mathrm{ABC}$ to intensify the search region.

Now a day a number of researchers are moving towards ABC algorithm from all over the world. A lot of modifications are done in $\mathrm{ABC}$ in recent years after its inception by $\mathrm{D}$. Karaboga [7]. In [10], D. Karaboga appears with an enhanced version of $\mathrm{ABC}$ for constrained optimization problems. He applied it in neural network training [11], to classification of medical pattern and clustering problems [12] and to solve TSP problems [13]. Artificial Bee Colony algorithm applied in Distributed Environments by A. Banharnsakun et al. [14]. Harish Sharma et al. developed DSABC [15], Balanced ABC [16], MeABC [17], and LFABC [18]. S. Pandey et al. developed a hybrid of $\mathrm{ABC}$ using crossover operation and applied it on TSP [19]. A survey of ABC carried out by $\mathrm{H}$. Sharma et al. [20] shows its popularity in last decade.

R. Ramanathan used $\mathrm{ABC}$ algorithm in image compression [21]. A. Ahmad et al.[22] employed artificial bee colony (ABC) algorithm to formulate uniformly excited, nonuniformly spaced, symmetrical linear arrays. ABC algorithm is used to minimize the side lobe level (SLL) of uniformly excited linear antenna arrays by considering the element 
spacing as the optimization parameters. Alvarado Iniesta [23] makes use of $\mathrm{ABC}$ algorithm to optimize the flow of material in a manufacturing plant. They examine how to optimize the time and effort required to supply raw material to separate production lines in a manufacturing plant by minimizing the distance an operator must travel to distribute material from a warehouse to a set of different production lines with corresponding demand. A Ozen [24] developed a new artificial bee colony algorithm based modulation recognition technique for multipath fading channels.

The organization of this paper as follow: Section 2 gives brief idea about original $\mathrm{ABC}$, analogy between behavior of honey bees and artificial bee colony algorithm. Section 3 introduces linear crossover operator. Section 4 outlines a new hybrid algorithm that integrates $\mathrm{ABC}$ and GA. Next section shows what are experimental setup and results for 4 benchmark functions. A real world problem taken in section 6 and proposed algorithm tested for it. Conclusion of this paper presented in section 7 followed by appendix with figures and references.

\section{ARTIFICIAL BEE COLONY ALGORITHM}

Artificial Bee Colony Algorithm is motivated from the intelligent food foraging behavior of honey bee insects. Honey bee swarm is one of the most intelligent swarms exists in nature; which follows collective intelligent method, while searching the food. The honey bee swarm has many qualities like bees can communicate the information, can memorize the environment, can store and share the information and take decisions based on that. According to changes in the environment, the swarm updates itself, assigns the tasks dynamically and moves further by social learning and teaching. This intelligent behavior of bees motivates researchers to simulate above foraging behavior of the bee swarm.

\subsection{Analogy of Artificial Bee Colony Algorithm}

The original model proposed by D. Karaboga [7] is composed of three major elements: employed and unemployed foragers, and food sources. The employed bees are ally with an appropriate food source. Employed bees have intimate knowledge about food source. Exploitation of food sources done by employed bees. When a food source abandoned employed bee become unemployed. The unemployed foragers are bees having no information about food sources and searching for a food source to exploit it. One can classify unemployed bees in two categories: scout bees and onlooker bees. Scout bees search at random for new food sources surrounding the hive. Onlooker bees observe the waggle dance in hive, to select a food source for exploitation. The third element is the rich food sources close to their hive. Comparatively in the optimization context, the number of food sources (that is the employed or onlooker bees) in $\mathrm{ABC}$ algorithm, is equivalent to the number of solutions in the population. Moreover, the location of a food source represents the position of a favorable solution to the optimization problem, since the trait of nectar of a food source represents the fitness cost (quality) of the correlated solution.

\subsection{Phases of Artificial Bee Colony Algorithm}

The search process of $\mathrm{ABC}$ follow three major steps [7]:

- Send the employed bees to a food source and calculate the nectar quality;

- Onlooker bees select the food sources after gathering information from employed bees and calculating the nectar quality;

- Determine the scout bees and employ them onto possible food sources.

The location of the food sources are arbitrarily selected by the bees at the initial stage and their nectar qualities are measured. The employed bees then share the nectar information of the sources with the onlooker bees waiting at the dance area within the hive. After sharing this information, each employed bee returns to the food source checked during the previous cycle, as the location of the food source had been recalled and then selects new food source using its observed information in the neighborhood of the present food source. At the last stage, an onlooker bee uses the information retrieved from the employed bees at the dance area to select a good food source. The possibility for the food sources to be elected boosts with boost in its quality of nectar. Hence, the employed bee with information of a food source with the highest quality of nectar employs the onlookers to that food source. It afterward chooses another food source close by the one presently in her memory depending on observed information. A new food source is randomly generated by a scout bee to replace the one abandoned by the onlooker bees. This complete search process could be outlined in Fig. 1 as follows [7]:

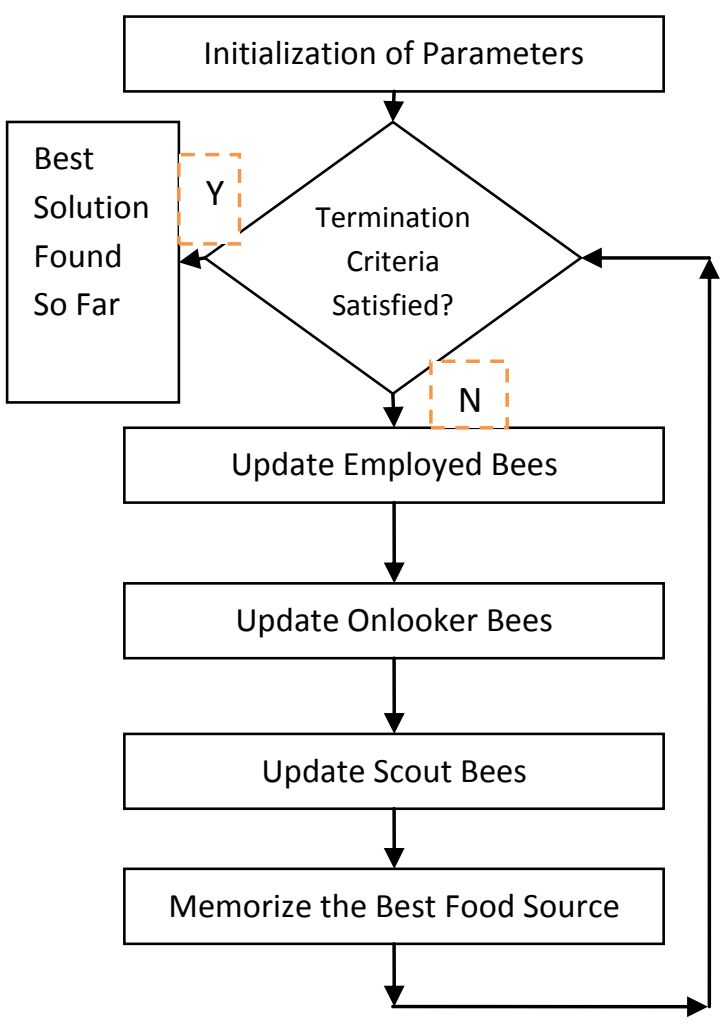

Fig 1: Phases in Artificial Bee Colony 


\subsubsection{Initialization of Swarm}

The ABC algorithm has three parameters: the number of food sources (population), the number of test after which a food source is treated to be jilted (limit) and the termination criteria (maximum number of cycle). In the original $\mathrm{ABC}$ proposed by D. Karaboga [7], the number of food sources is equal to the employed bees or onlooker bees. Initially it consider an evenly dealt swarm of food sources (SN), where each food source $x_{i}(i=1,2 \ldots S N)$ is a D-dimensional vector. Each food source is generated using following method [8]:

$$
x_{i j}=x_{\operatorname{minj}}+\operatorname{rand}[0,1]\left(x_{\max j}-x_{\operatorname{minj}}\right)
$$

Where

- $\operatorname{rand}[0,1]$ is a function that generates an evenly distributed random number in range $[0,1]$.

\subsubsection{Employed Bee}

Employed bees phase update the present solution based on the information of individual experiences and the fitness value of the newly found solution. New food source with higher fitness value replace the existing one. The position update equation for $\mathrm{j}^{\text {th }}$ dimension of $\mathrm{i}^{\text {th }}$ candidate during this phase is shown below [8]:

$V_{i j}=x_{i j}+\varphi_{i j}\left(x_{i j}-x_{k j}\right)$

Where

- $\varphi_{i j}\left(x_{i j}-x_{k j}\right)$ is known as step size, $\mathrm{k} \in\{1,2, \ldots$, $\mathrm{SN}\}, \mathrm{j} \in\{1,2, \ldots, \mathrm{D}\}$ are two randomly chosen indices. $\mathrm{k} \neq \mathrm{i}$ ensure that step size has some indicative improvement.

\subsubsection{Onlooker Bee}

The number of food sources for onlooker bee is same as the employed. During this phase all employed bee share fitness information of new food sources with onlooker bees. Onlooker bees calculate the selection probability of each food source generated by the employed bee. The best fittest food source is selected by the onlooker. There are number of method for calculation of probability, but it must include fitness. Probability of each food source is decided using its fitness as follow [8]:

$P_{i}=\frac{\text { fit }_{i}}{\sum_{i=1}^{S N} f_{i t}}$

\subsubsection{Scout Bee Phase}

If the location of a food source is not updated for a predefined number of cycles, then the food source is assumed to be neglected and scout bees phase is initialized. During this phase the bee associated with the neglected food source converted into scout bee and the food source is replaced by the arbitrarily chosen food source inside the search space. In $\mathrm{ABC}$, the predefined number of cycles is an important control parameter which is called limit for rejection. Now the scout bees replace the abandoned food source with new one using following equation [8].

$$
\begin{gathered}
x_{i j}=x_{\min j}+\operatorname{rand}[0,1]\left(x_{\operatorname{maxj}}-x_{\operatorname{minj}}\right) \\
\forall j=1,2, \ldots \ldots D
\end{gathered}
$$

Based on the above description, it is clear that in $\mathrm{ABC}$ search process there are three main control parameters: the number of food sources SN (same as number of onlooker or employed bees), the limit and the maximum number of cycles. The algorithm of the $\mathrm{ABC}$ is outlined as follow [8]:

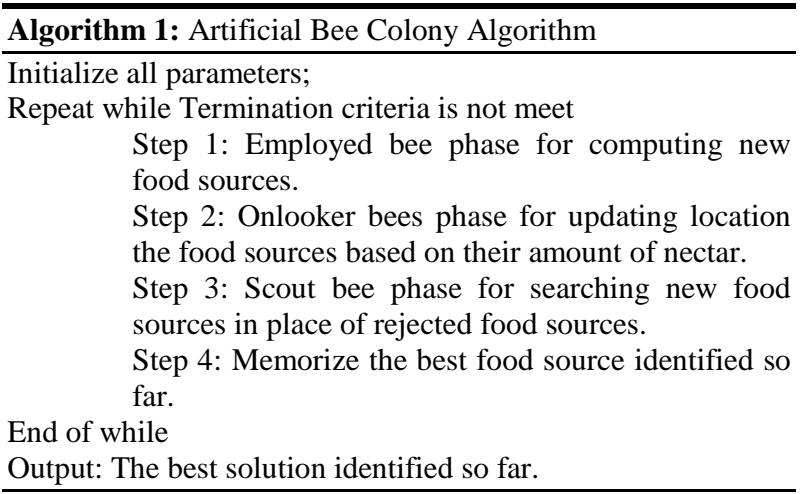

\section{CROSSOVER OPERATOR}

The crossover operator is a method for earning genetic information from parents; it combines the features of two parents to form two off-springs, with the probability that good chromosomes may evaluate better ones. The crossover operator is not regularly enforced to all pairs of parent solution the intermediate generation. An incidental choice is made, where the plausibility of crossover being applied depends on probability determined by a crossover rate, known as crossover probability. The crossover operator is most important part in GAs. It combines fraction of good solution to construct new favorable solution. Information involved in one solution mixed with information involved in another solution and the emerging solution will either have good fitness or survive to commutate this information again. If generated two off-springs are identical then crossover operator show strong heritability $[25,26]$.

\subsection{Real Coded Crossover Operators}

Crossover operators play major role in genetic algorithm which combines the feature of existing solutions and generate new solutions. The optimization problems depend upon the data they used so they are classified in to two categories. One is based on real data set and another one is based on binary or discrete data set. Crossover operator also categorized as binary crossover operators and real coded crossover operators. This paper used the real coded crossover operator for experiment. J C Bansal et al. developed a methodology to share information between two particles using a laplacian operator designed from Laplace probability density function. In this strategy two particles share their positional information in the search space and a new particle is formed. The particle, is known as laplacian particle, replaces the worst performing particle in the swarm. Using this new operator, this paper introduces two algorithms namely Laplace Crossover PSO with inertia weight (LXPSO-W) and Laplace Crossover PSO with constriction factor (LXPSO-C) [27]. A. H. Wright suggests a genetic algorithm that uses real parameter vectors as chromosomes, real parameters as genes, and real numbers as alleles [28].

\subsubsection{Linear Crossover}

Linear crossover $[27,28]$ is one of the earliest operator in real coded crossover it develops three solutions from two parents and the best two o springs replace parents. Let $\left(x_{1}^{(1, t)} x_{2}^{(1, t)}\right.$ $\left.x_{3}^{(1, t)} x_{4}^{(1, t)} \ldots x_{n}^{(1, t)}\right)$ and $\left(x_{1}^{(2, t)} x_{2}^{2, t)} x_{3}^{(2, t)} x_{4}^{(2, t)} \ldots x_{n}^{(2, t)}\right)$ are two parent solutions of dimension $n$ at generation $t$. Linear crossover develops three offspring from these parents as 
shown in Eq.(5, 6 and 7) and best two offspring being chosen as off-springs.

$$
\begin{aligned}
& 0.5\left(\mathrm{x}_{\mathrm{i}}^{(1, \mathrm{t})}+\mathrm{x}_{\mathrm{i}}^{(2, \mathrm{t})}\right) \\
& \left(1.5 \mathrm{x}_{\mathrm{i}}^{(1, \mathrm{t})}-0.5 \mathrm{x}_{\mathrm{i}}^{(2, \mathrm{t})}\right) \\
& \left(-0.5 \mathrm{x}_{\mathrm{i}}^{(1, \mathrm{t})}+1.5 \mathrm{x}_{\mathrm{i}}^{(2, \mathrm{t})}\right)
\end{aligned}
$$

Where $\mathrm{i}=1,2, \ldots, \mathrm{n}$

\section{PROPOSED HYBRID CROSSOVER BASED ARTIFICIAL BEE COLONY (CbABC) ALGORITHM}

$\mathrm{ABC}$ with crossover works in five different phases: first phase is initialization of parameters; second phase is employed bee phase to compute new food sources. Third phase is newly introduces crossover phase. This phase maintains balance between diversification and intensification. Crossover phase diversify the population. Fourth phase is onlooker bee phase to improve solution based on their fitness. Last phase is scout bee phase, this phase search new solutions in place of rejected solutions.

The algorithm of crossover based artificial bee colony $(\mathrm{CbABC})$ algorithm outlined as follow:

Algorithm 2: Hybrid Crossover Based Artificial Bee Colony (CbABC) Algorithm

Initialize all the parameters;

Repeat while Termination criteria is not meet

Step 1: Employed bee phase for computing new food sources.

Step 2: Crossover phase to increase the quality of solution

Step 3: Onlooker bees phase for updating the location of food sources based on their nectar amounts.

Step 4: Scout bee phase for searching new food sources in place of rejected food sources.

Step 5: Memorize the best food source identified so far.

End of while loop

Output: Best solution identified so far.

The first step consists of the evaluation of the population using the Artificial Bee Colony. Initial populations generated by $\mathrm{ABC}$ are used by employed bees. After this crossover operators are applied. If crossover criteria or probability fulfilled than two random parents are taken to perform crossover operation on them. After crossover operation new off-springs are developed. Worst parent replaced by best developed offspring if its fitness is better than the worst parent. Crossover operator is applied to two arbitrarily selected parents from current population. Two offspring developed from crossover and worst parent is replaced by best offspring, other parent remains same. The complete process repeats itself until the maximum numbers of cycles are completed. The phases of modified $\mathrm{ABC}$ algorithm are outlined in Fig. 2.

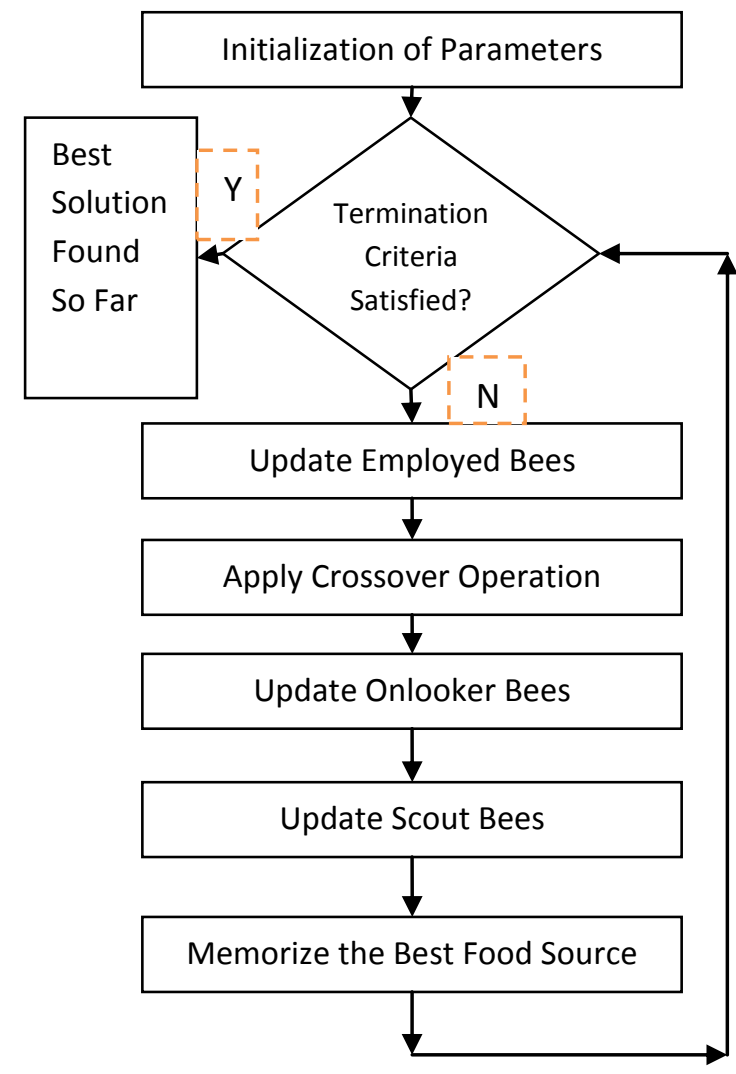

Fig 2: Phases in Hybrid Crossover based Artificial Bee Colony (CbABC) Algorithm

In this paper crossover applied in each iteration after employed bee phase. The detailed pseudo code of proposed crossover based $\mathrm{ABC}$ algorithm is outlined in algorithm 3 .

Algorithm 3: Detailed Hybrid Crossover Based ABC (CbABC) Algorithm

Initialization phase

For $i=0$ to the maximum no. of food source size do

For $j=0$ to the problem dimension

Initialize all food sources arbitrarily using equation (1)

End for $j$

Evaluate fitness for all food sources

End for $i$

Re pe at

Employed Bee Phase

For $i=0$ to maximum no. of employed bees

For $j=0$ to maximum problem dimension

Generate new candidate solution using equation (2)

End for $j$

Compute fitness values for all new generated candidate solution

If candidate solution is better than the old solution then replace old solution with candidate solution

End for $i$

Crossover phase

If crossover criteria satisfies

For $i=0$ to the maximum no. of food source

Select two random individuals from the current population for crossover operation.

Apply crossover operation.

New off-springs generated from parents as a result of crossover. Replace the worst parent with the best new 
End for $i$

Onlooker Bee phase

For $i=0$ to the maximum no. of onlooker bees

For $j=0$ to maximum dimension

Generate new candidate solution

End for $j$

Calculate the fitness for new generated candidate solution If candidate solution has the better fitness values then replace old solution

End for $i$

Scout Bee phase

If any food source exhausted

Initialize randomly exhausted food source until maximum cycle no.

\section{EXPERIMENTAL RESULTS}

\subsection{Test problems under consideration}

Artificial Bee Colony algorithm with linear crossover applied to the four benchmark functions for whether it gives better result or not at different probability. Benchmark functions taken in this paper are of different characteristics like unimodel (Sphere, Rosenbrock) or multi-model (Griewank, Rastrigin) and separable (Sphere, Rastrigin) or non-separable (Griewank, Rosenbrock). In order to analyze the performance of $\mathrm{CbABC}, 4$ different global optimization problems $\left(f_{1}\right.$ to $\left.f_{4}\right)$ are selected (listed in Table 1). These are continuous optimization problems and have different degrees of complexity and multimodality. Test problems $f_{l}-f_{4}$ are taken from [14].

Table 1. Test problems

\begin{tabular}{|c|c|c|c|}
\hline $\begin{array}{l}\text { Test } \\
\text { Problem }\end{array}$ & Objective function & $\begin{array}{l}\text { Characteris } \\
\text { tic }\end{array}$ & Ranges \\
\hline Sphere & $f_{1}(\vec{x})=\sum_{i=1}^{D} x_{i}^{2}$ & $\begin{array}{l}\text { Uni-model, } \\
\text { Separable }\end{array}$ & $\begin{array}{l}-100 \leq x_{i} \\
\leq 100\end{array}$ \\
\hline $\begin{array}{l}\text { Griew- } \\
\text { ank }\end{array}$ & $\begin{array}{l}=\frac{1}{4000}\left(\sum_{i=1}^{D}\left(x_{i}^{2}\right)\right) \\
-\left(\prod^{D} \cos \left(\frac{x_{i}}{r}\right)\right)+1\end{array}$ & $\begin{array}{l}\text { Multi- } \\
\text { model, } \\
\text { Non- } \\
\text { separable }\end{array}$ & $\begin{array}{l}-600 \leq x_{i} \\
\leq 600\end{array}$ \\
\hline $\begin{array}{l}\text { Rastrig- } \\
\text { in }\end{array}$ & $\begin{array}{l}f_{3}(\vec{x})=\sum_{i=1}^{D}\left(x_{i}^{2}-\right. \\
\left.10 \cos \left(2 \pi x_{i}\right)+10\right)\end{array}$ & $\begin{array}{l}\text { Multi- } \\
\text { model, } \\
\text { Separable }\end{array}$ & $\begin{array}{l}-5.12 \leq x_{i} \\
\leq 5.12\end{array}$ \\
\hline $\begin{array}{l}\text { Rosenb- } \\
\text { rock }\end{array}$ & $\begin{array}{l}f_{4}(\vec{x}) \\
=\sum_{i=1}^{D} 100\left(x_{i}^{2}\right. \\
\left.-x_{i+1}\right)^{2}+\left(1-x_{i}\right)^{2}\end{array}$ & $\begin{array}{l}\text { Uni-model, } \\
\text { Non- } \\
\text { separable }\end{array}$ & $\begin{array}{l}-30 \leq x_{i} \\
\leq 30\end{array}$ \\
\hline
\end{tabular}

\subsection{Experimental Setup}

To prove the efficiency of $\mathrm{CbABC}$, it is compared with original $\mathrm{ABC}$ over considered four problems, following experimental setting is adopted:

- $\quad$ The size of colony= Population size $\mathrm{SN}=20$

- Number of Employed bee $=$ Number of Onlooker bee $=\mathrm{SN} / 2=10$
- The maximum number of cycles for foraging $\mathrm{MCN}$ $=2000$

- Number of repetition of experiment $=$ Runtime $=30$

- Dimension of Problem space $\mathrm{D}=30$

- Limit $=100$,A food source which could not be improved through "limit" trial is abandoned by it employed bee

- 4 Benchmark functions are used and each has minimum value $=0$

- The mean function values of the best solutions found by the algorithms for different dimensions have been recorded.

\subsection{Result Comparison}

Results with experimental setting discussed above are outlined in table 2. In table 2 mean objective (MO) and average evaluation (AE) are reported. In this paper original ABC compared with linear crossover (LX). Table 2 shows that most of the time Crossover based $\mathrm{ABC}$ outperformed original $\mathrm{ABC}$ in terms of performance.

Table 2 Comparison of the results of Test problems

\begin{tabular}{ccccc}
\hline $\begin{array}{c}\text { Test } \\
\text { Function }\end{array}$ & Measure & ABC & Pr & LX
\end{tabular}

\begin{tabular}{|c|c|c|c|c|}
\hline \multirow{6}{*}{$f$} & \multirow{3}{*}{ MO } & \multirow{3}{*}{$4.589 \mathrm{e}-02$} & 0.1 & $4.596 \mathrm{e}-02$ \\
\hline & & & 0.2 & $4.612 \mathrm{e}-02$ \\
\hline & & & 0.3 & $4.565 \mathrm{e}-02$ \\
\hline & \multirow{3}{*}{$\mathrm{AE}$} & \multirow{3}{*}{2167} & 0.1 & 2296 \\
\hline & & & 0.2 & 2248 \\
\hline & & & 0.3 & 2386 \\
\hline \multirow{6}{*}{$f_{2}$} & \multirow{3}{*}{ MO } & & 0.1 & $2.548 \mathrm{e}-06$ \\
\hline & & \multirow{2}{*}{$2.417 \mathrm{e}-06$} & 0.2 & $2.561 \mathrm{e}-06$ \\
\hline & & & 0.3 & $2.428 \mathrm{e}-06$ \\
\hline & \multirow{3}{*}{$\mathrm{AE}$} & \multirow{3}{*}{5137} & 0.1 & 5190 \\
\hline & & & 0.2 & 5307 \\
\hline & & & 0.3 & 5567 \\
\hline \multirow{6}{*}{$f_{3}$} & \multirow{3}{*}{ MO } & \multirow{3}{*}{276.3} & 0.1 & 271.6 \\
\hline & & & 0.2 & 277.2 \\
\hline & & & 0.3 & 279.7 \\
\hline & \multirow{3}{*}{$\mathrm{AE}$} & \multirow{3}{*}{20000} & 0.1 & 20000 \\
\hline & & & 0.2 & 20000 \\
\hline & & & 0.3 & 20000 \\
\hline$f_{4}$ & MO & & 0.1 & 9.546 \\
\hline
\end{tabular}


9.816 $0.2 \quad 9.582$

$0.3 \quad 9.715$

$0.1 \quad 20000$

$\mathrm{AE}$ $0.2 \quad 20000$ 20000
Obtained results indicate that $\mathrm{ABC}$ With appropriate crossover and appropriate crossover probability may provide far better results than original ABC. However there is no fixed value of crossover probability for which crossover operator can improve the results generated by $\mathrm{ABC}$. However, from the applications point of view a range from 0.1 to 0.3 is the most suitable for crossover probability as shown by tables, crossover operator when applied to basic $\mathrm{ABC}$ algorithm improves the results in terms of performance and accuracy. $\mathrm{CbABC}$ algorithm improves the results of TSP as compared to $\mathrm{ABC}$ algorithm.

Table 2 lists the comparison results of these operators for 4 benchmark functions of 3 different probabilities for $\mathrm{ABC}$ algorithm. Each of experiments was repeated 30 times, and the mean objective (MO) and average evaluation (AE) are listed in the tables, of which the bold mean values are the best results among the listed methods. From the above table it can be observed that with the probability increasing, the mean objective values of most of the benchmark functions are decreased. For almost all functions most of the time Crossover based $\mathrm{ABC}$ outperformed original $\mathrm{ABC}$ in terms of performance..

\section{APPLICATION OF PROPOSED ALGORITHM}

To measure the robustness of the proposed strategy, a real world optimization problem, namely travelling salesman problem considered. The Traveling Salesman Problem (TSP) is one of the hardest problems for which no efficient method is known. Assuming a travelling salesman has to travel a given number of cities, starting and ending at the same city. This tour, which represents the length of the travelled path, is the formulation of TSP. As the number of cities increases, the determination of the optimal tour (also known as Hamiltonian tour), becomes very complex. A TSP decision problem is generally classified as NP-Complete problem.

The TSP can be defined on a complete undirected graph $G=$ $(V, E)$ if it is symmetric or on a directed graph $G=(V, A)$ if it is asymmetric. Here $\mathrm{V}, \mathrm{E}$ and $\mathrm{A}$ are set of vertices, edges and arcs respectively. The set $\mathrm{V}=\{1, \ldots, \mathrm{n}\}$ is the vertex set, $E=$ $\{(i, j): i, j \in V, i<j\}$ is an edge set and $A=\{(i, j): i, j \in V, i \neq$ $j\}$ is an arc set. A cost matrix $\mathrm{C}=\left(c_{i j}\right)$ is defined either on $\mathrm{E}$ or on A. The cost matrix satisfies the triangle inequality whenever $c_{i j} \leq c_{i k}+c_{k j}$, for all values of $i, j$, $k$. specially, this is the case of planar problems for which the vertices are points $P_{i}=\left(\mathrm{x}_{i}, \mathrm{y}_{i}\right)$ in the plane, and $c_{i j}=\sqrt{\left(x_{i}-x_{j}\right)^{2}+\left(y_{i}-y_{j}\right)^{2}}$ is known as the Euclidean distance. The triangle inequality is also satisfied if $c_{i j}$ is the length of a shortest path from $i$ to $j$ on graph $G$.
Experimental setup for finding solution of TSP is same as it was for standard benchmark function except that now it is tested for different number of cycles. Number of cycles varies from 500 to 3000 . CbABC use linear crossover operator for solving travelling salesman problem.

\subsection{Experimental Results}

The $\mathrm{CbABC}$ using linear crossover operator outperformed ordinary ABC. Results are taken for dimension 10, 20 and 30 with varying maximum cycle numbers (from 500 to 3000 ). Table 3 summarizes these results. Simple ABC algorithm degrades performance when maximum cycle number exceeds 1500 but $\mathrm{CbABC}$ continuously keeps improving results.

Table 3 Comparison of the results for Travelling Salesman problem

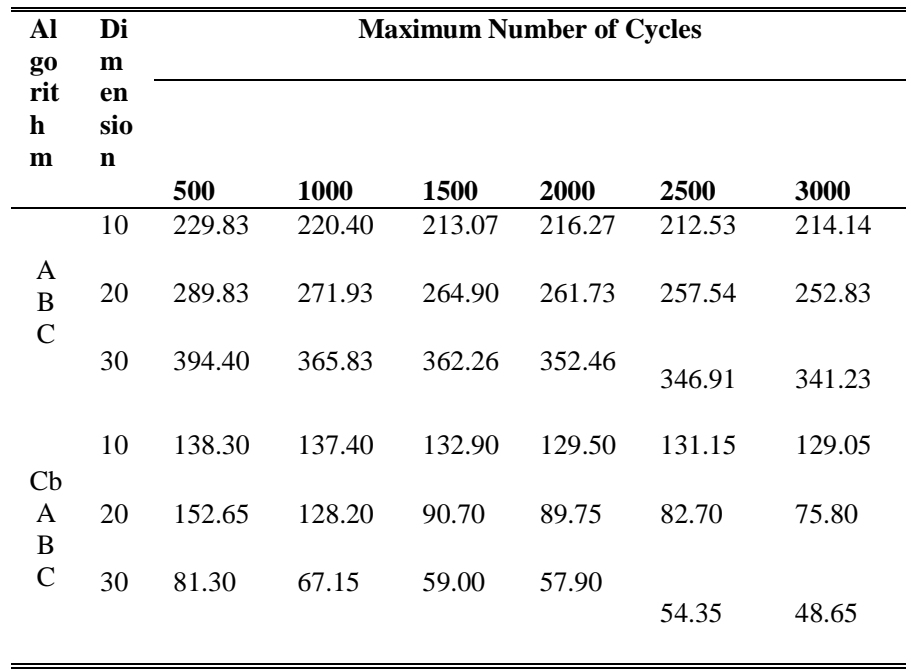

The proposed algorithm compared on the basis of dimension and maximum number of cycles. The $\mathrm{CbABC}$ algorithm improves the results of TSP as compared to ABC algorithm. CbABC improves result, when dimension increased. Fig.4-6 shows that how $\mathrm{CbABC}$ outperformed simple $\mathrm{ABC}$ when applied for TSP.

\section{CONCLUSION}

This work presents a novel hybrid optimization algorithm named $\mathrm{CbABC}$. This algorithm integrates crossover operator from GA with $\mathrm{ABC}$. Linear crossover operator is applied to the Artificial Bee Colony algorithm. The worst parent replaced by an off-spring generated by two randomly selected parents in each cycle, if fitness of generated off-spring is better than the worst parent from the current swarm. The experiments are performed on a set of four well known benchmark functions. Crossover probability range [0.1 -0.3$]$ considered for experiments. It is observed that linear crossover operator with $\mathrm{ABC}$ is better choice for continuous optimization. Performance of proposed algorithm refined with increment in crossover probability. It also shows that $\mathrm{CbABC}$ algorithm improves the TSP results in comparison to original $\mathrm{ABC}$ algorithm. Numerical results show that the proposed method is superior to ABC approach. Proposed algorithm has the ability to get out of a local minimum and can be efficiently used for separable, multivariable, multimodal function optimization. 


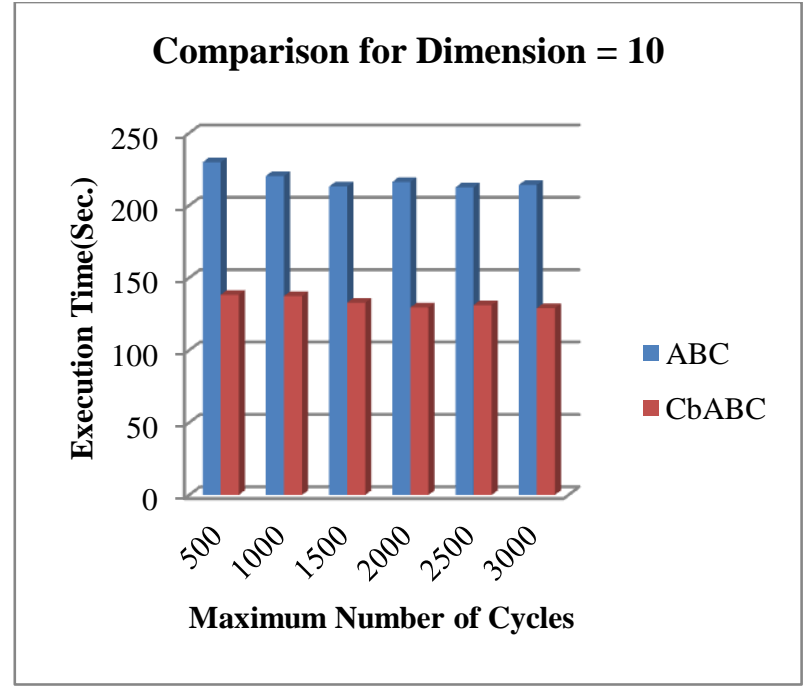

Fig 3: Comparison of $\mathrm{ABC}$ and $\mathrm{CbABC}$ for $\mathrm{TSP}$ at $\mathrm{D}=10$

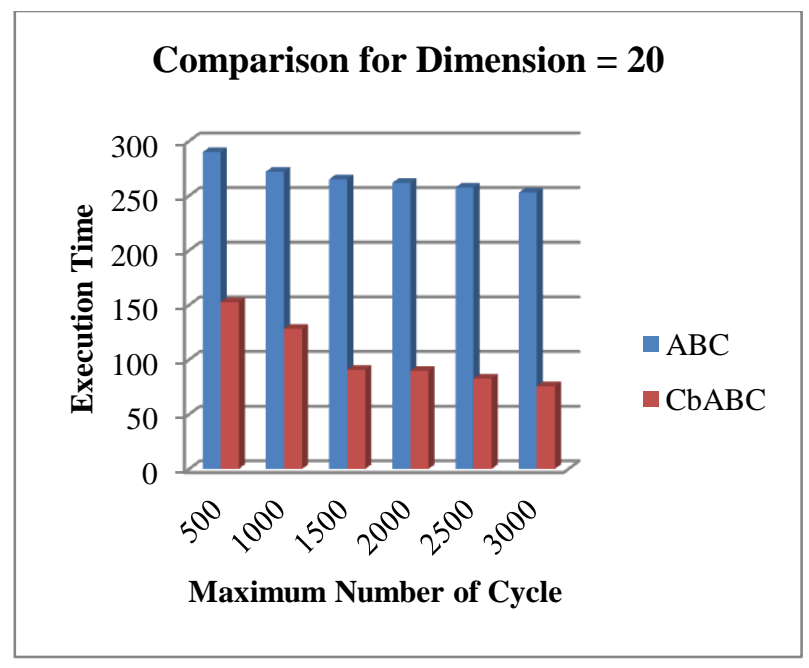

Fig 4: Comparison of $\mathrm{ABC}$ and $\mathrm{CbABC}$ for $\mathrm{TSP}$ at $\mathrm{D}=\mathbf{2 0}$

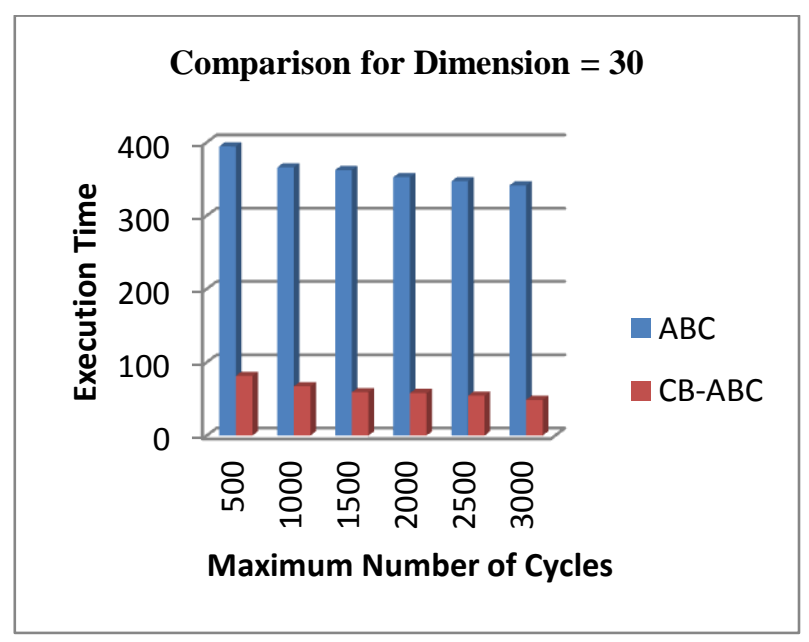

Fig 5: Comparison of $\mathrm{ABC}$ and $\mathrm{CbABC}$ for $\mathrm{TSP}$ at $\mathrm{D}=\mathbf{3 0}$

\section{REFERENCES}

[1] X.S. Yang. Nature-inspired metaheuristic algorithms. Luniver Press, 2011.

[2] M. Dorigo et al. "Ant colony optimization: a new metaheuristic". In Evolutionary Computation, 1999. CEC 99. Proceedings of the 1999 Congress, volume 2. IEEE, 1999.

[3] J. Kennedy et al. "Particle swarm optimization. In Neural Networks, 1995". Proceedings, IEEE International Conference on, volume 4, pages 1942-1948. IEEE, 1995.

[4] K.V. Price et al. "Differential evolution: a practical approach to global optimization". Springer Verlag, 2005.

[5] J. Vesterstrom et al. "A comparative study of differential evolution, particle swarm optimization, and evolutionary algorithms on numerical benchmark problems". In Evolutionary Computation, 2004. CEC2004. Congress on, volume 2, pages 1980-1987. IEEE, 2004.

[6] K.M. Passino. "Biomimicry of bacterial foraging for distributed optimization and control". Control Systems Magazine, IEEE, 22(3):52-67, 2002.

[7] D. Karaboga. "An idea based on honey bee swarm for numerical optimization". Techn. Rep. TR06, Erciyes Univ. Press, Erciyes, 2005.

[8] D. Karaboga et al. "A comparative study of artificial bee colony algorithm". Applied Mathematics and Computation, 214(1):108-132, 2009.

[9] G. Zhu et al. "Gbest-guided artificial bee colony algorithm for numerical function optimization". Applied Mathematics and Computation, 217(7):3166-3173, 2010.

[10] D. Karaboga et al. "Artificial bee colony (abc) optimization algorithm for solving constrained optimization problems". Foundations of Fuzzy Logic and Soft Computing, pages 789-798, 2007.

[11] D. Karaboga, et al. "Artificial bee colony (abc) optimization algorithm for training feed-forward neural networks". Modeling decisions for artificial intelligence, pages 318-329, 2007.

[12] B. Akay et al. "Training neural networks with abc optimization algorithm on medical pattern classification". International Conference on Multivariate Statistical Modelling and High Dimensional Data Mining (Kayseri, TURKEY), June 1923, 2008.

[13] F. Xing et al. "The parameter improvement of bee colony algorithm in tsp problem". Science Paper Online, November 2007.

[14] A. Banharnsakun et al. "Artificial bee colony algorithm on distributed environments". In Nature and Biologically Inspired Computing (NaBIC), 2010 Second World Congress on, pages 13-18. IEEE.

[15] Harish Sharma et al. "Dynamic Swarm Artificial Bee Colony Algorithm." IJAEC 3.4 (2012): 19-33. Web. 5 Aug. 2013. doi:10.4018/jaec.2012100102

[16] Bansal, Jagdish Chand, et al. "Balanced artificial bee colony algorithm." International Journal of Artificial Intelligence and Soft Computing 3.3 (2013): 222-243. 
[17] Bansal, Jagdish Chand, et al. "Memetic search in artificial bee colony algorithm." Soft Computing (2013): $1-18$.

[18] Harish Sharma et al. "Opposition based lévy flight artificial bee colony." Memetic Computing (2012): 1-15.

[19] Pandey, Shailesh et al. "Enhanced Artificial Bee Colony Algorithm and It's Application to Travelling Salesman Problem." HCTL Open International Journal of Technology Innovations and Research, Volume 2, (2013):137-146, ISSN: 2321-1814, ISBN: 978-1-62776111-6.

[20] Bansal, Jagdish Chand et al. "Artificial bee colony algorithm: a survey." International Journal of Advanced Intelligence Paradigms 5.1 (2013): 123-159.

[21] Ramanathan, R., K. Kalaiarasi, and D. Prabha. "Improved wavelet based compression with adaptive lifting scheme using Artificial Bee Colony algorithm." International Journal of Advanced Research in Computer Engineering \& Technology (IJARCET) 2.4 (2013): pp-1549.

[22] Ahmad, Aamir, et al. "Artificial bee colony algorithm to reduce the side lobe level of uniformly excited linear antenna arrays through optimized element spacing."
Information \& Communication Technologies (ICT), 2013 IEEE Conference on. IEEE, 2013: pp 1029-1032.

[23] Alvarado-Iniesta, Alejandro, et al. "Optimization of the material flow in a manufacturing plant by use of artificial bee colony algorithm." Expert Systems with Applications (2013).

[24] Ozen, Ali, and Celal Ozturk. "A novel modulation recognition technique based on artificial bee colony algorithm in the presence of multipath fading channels." Telecommunications and Signal Processing (TSP), 2013 36th International Conference on. IEEE, 2013.

[25] J. H. Holland. "Outline for a logical theory of adaptive systems". Journal of the ACM, 3:297-314, 1962.

[26] Talbi, El-Ghazali. "Metaheuristics: from design to implementation”. Vol. 74. John Wiley \& Sons, 2009.

[27] Bansal, J. C. et al. "Information Sharing Strategy among Particles in Particle Swarm Optimization Using Laplacian Operator", Swarm Intelligence Symposium, 2009. IEEE, pages 30-36.

[28] Wright, “A. Genetic Algorithms for Real Parameter Optimization, Foundations of Genetic Algorithms", G. Rswlins(Ed.), Morgen Kaufmann publishers, CA, 1991, pp. 205-218. 